\title{
Experiments and simulations of MEMS thermal sensors for wall shear-stress measurements in aerodynamic control applications
}

\author{
Qiao Lin ${ }^{1}$, Fukang Jiang ${ }^{2}$, Xuan-Qi Wang ${ }^{3}$, Yong $\mathbf{X u}^{4}$, \\ Zhigang $\mathrm{Han}^{3}$, Yu-Chong Tai ${ }^{3}$, James Lew ${ }^{5}$ and Chih-Ming $\mathrm{Ho}^{5}$ \\ ${ }^{1}$ Department of Mechanical Engineering, Carnegie Mellon University, Pittsburgh, \\ PA 15213, USA \\ ${ }^{2}$ Umachines, Altadena, CA 91001, USA \\ ${ }^{3}$ Division of Engineering and Applied Science, California Institute of Technology, Pasadena, \\ CA 91125, USA \\ ${ }^{4}$ Department of Electrical and Computer Engineering, Wayne State University, Detroit, \\ MI 48202, USA \\ ${ }^{5}$ Department of Mechanical and Aerospace Engineering, University of California, \\ Los Angeles, CA 90024, USA \\ E-mail: qlin@andrew.cmu.edu
}

Received 19 March 2004, in final form 22 June 2004

Published 26 August 2004

Online at stacks.iop.org/JMM/14/1640

doi:10.1088/0960-1317/14/12/007

\begin{abstract}
MEMS thermal shear-stress sensors exploit heat-transfer effects to measure the shear stress exerted by an air flow on its solid boundary, and have promising applications in aerodynamic control. Classical theory for conventional, macroscale thermal shear-stress sensors states that the rate of heat removed by the flow from the sensor is proportional to the $1 / 3$-power of the shear stress. However, we have observed that this theory is inconsistent with experimental data from MEMS sensors. This paper seeks to develop an understanding of MEMS thermal shear-stress sensors through a study including both experimental and theoretical investigations. We first obtain experimental data that confirm the inadequacy of the classical theory by wind-tunnel testing of prototype MEMS shear-stress sensors with different dimensions and materials. A theoretical analysis is performed to identify that this inadequacy is due to the lack of a thin thermal boundary layer in the fluid flow at the sensor surface, and then a two-dimensional MEMS shear-stress sensor theory is presented. This theory incorporates important heat-transfer effects that are ignored by the classical theory, and consistently explains the experimental data obtained from prototype MEMS sensors. Moreover, the prototype MEMS sensors are studied with three-dimensional simulations, yielding results that quantitatively agree with experimental data. This work demonstrates that classical assumptions made for conventional thermal devices should be carefully examined for miniature MEMS devices. (Some figures in this article are in colour only in the electronic version)
\end{abstract}




\section{Introduction}

When a fluid flows over a solid surface, viscous effects generate shear stress, or skin friction, on the surface. Knowledge of such wall shear stress is essential for understanding the dynamics of fluid flow, and its measurement holds great importance for investigating and controlling wall-bound turbulence and flow separation in aerodynamic control [1-5]. A large number of shear-stress measurement instruments have been developed, which are either based on direct methods using a force transducer, or indirect methods that detect changes in physical parameters other than the force resulting from shear stress $[6,7]$.

Miniaturized shear-stress sensors fabricated using MEMS technology offer superior spatial resolution, fast time response and minimized interference with fluid flow. MEMS shearstress sensors can be based on direct methods, typically using micromachined force-sensitive floating elements [8-12]. These devices offer direct shear-stress measurement capabilities but are susceptible to contamination by dust and moisture from the medium. MEMS shear-stress sensors that exploit thermal effects generally do not suffer from this limitation. Thermally based shear-stress measurements are an indirect method, and there have been extensive developments of conventional thermal shear-stress measurement devices [13-16]. MEMS thermal shear sensors, in comparison, are miniaturized ( $\sim 200 \mu \mathrm{m}$ in dimensions) to allow maximal spatial resolution, minimal power consumption and negligible flow interference, and can be batch-fabricated inexpensively. Since their initial demonstration [17, 18], much progress has been made in further development of MEMS thermal sensors for shear-stress measurements. This includes prototypes with alternative sensing element configurations and materials [19-23], and investigations of sensor characteristics such as thermal insulation, frequency response, pressure sensitivity and noise floor spectra [24-26]. In addition, MEMS shear-stress sensors have been fabricated in arrays on rigid [27] and flexible substrates [28, 29], integrated with signal conditioning circuitry $[23,28,29]$ and applied to flow sensing and control in both air [30] and water [31]. Recently, there have also been numerical simulations on MEMS thermal shear-stress sensors to study the effect of natural convection on the fluid velocity profile [32] as well as the effect of heat conduction in the sensor substrate on frequency response [33].

Thermal shear-stress sensors exploit the dependence of heat transfer from a heated sensing element (or hot wire) on the applied shear stress and classical theory states that the rate at which heat is removed from the hotwire by the flow is proportional to the 1/3-power of the shear stress [7]. This theory has been applied to MEMS shear-stress sensors in the past due to the lack of a microscale sensor theory [21, 23, 26, 27]. However, we have observed that this law is often inconsistent with experimental data. This suggests that there may be phenomena that the classical theory does not consider, and that new understanding of the operation of MEMS thermal shear-stress sensors should be developed. This paper presents a study including both experimental and theoretical investigations to address this issue. By windtunnel testing of prototype MEMS shear-stress sensors with different geometric dimensions and materials, we first obtain experimental data that confirm the inadequacy of the classical theory. A theoretical analysis follows, identifying that this inadequacy is due to the lack of a thin thermal boundary layer in the fluid flow near the sensor surface. Then, a two-dimensional MEMS shear-stress sensor theory is developed. We show that by incorporating important heat-transfer effects that are ignored by the classical theory, the new model consistently explains the experimental data obtained from all prototype MEMS sensors tested. Moreover, we also present threedimensional simulations of the prototype MEMS sensors to gain a quantitative understanding of these devices. This work demonstrates that classical assumptions made for conventional thermal devices should be carefully examined for miniature MEMS devices.

\section{Sensor design and fabrication}

This section reviews the design and fabrication of MEMS thermal shear-stress sensors that were used in the experiments and simulations. A schematic of such sensors is illustrated in figure 1 [17, 23, 27]. The sensor consists of a polysilicon thin-film resistor on a silicon nitride or Parylene diaphragm, which in turn is suspended from the supporting substrate by a vacuum or air cavity. An electric current is passed (via aluminum metallization) through the resistor which functions as a hot wire. A vacuum or air cavity underneath the diaphragm is used to maximize thermal isolation. To measure shear stress exerted by a fluid flow on a solid wall, the sensor is mounted on the wall in such a way that the uncovered diaphragm surface is flush with the wall and the hot wire is perpendicular to the fluid velocity or shear stress which is proportional to the fluid velocity gradient at the wall. Since the heat transfer from the hot wire to the fluid flow is dependent upon the velocity profile, shear stress can be determined from the hot wire's thermal characteristics. Specifically, the measurement can be performed in constant-current mode, by passing a constant electric current through the hot wire and monitoring the hot wire temperature [7]. Alternatively, as is pursued in this paper, the measurement can occur in constant-temperature mode. In this case, feedback control is used to maintain the hot wire at a fixed temperature, and the electric power consumed in the hot wire is measured to obtain the shear stress.

The prototype sensors were fabricated using surfacemicromachining technology, and were based on either silicon nitride or Parylene diaphragms. The process for fabricating nitride-based sensors [17, 18, 27] began with a wafer that was coated with LPCVD silicon nitride. The nitride was RIE patterned to define the vacuum cavity, followed by an RIE etch of the exposed silicon. Local thermal oxidation was then performed with the nitride as a mask until the oxide grew to fill the nitride-silicon cavity. Next, a thin phosphosilicate glass (PSG) layer was deposited and patterned to form the etch channels. A second LPCVD silicon nitride layer was deposited, and patterned to open the etch holes. The cavity was then formed by removing the PSG/oxide using concentrated HF. A polysilicon layer was deposited, boron doped, annealed and RIE patterned to form the hot wire. The cavity was also sealed by this polysilicon layer. A fabricated nitride-based shear-stress sensor is shown in figure 2(a).

The fabrication of Parylene-based sensors, which were integrated with on-chip electronics, was based on a 


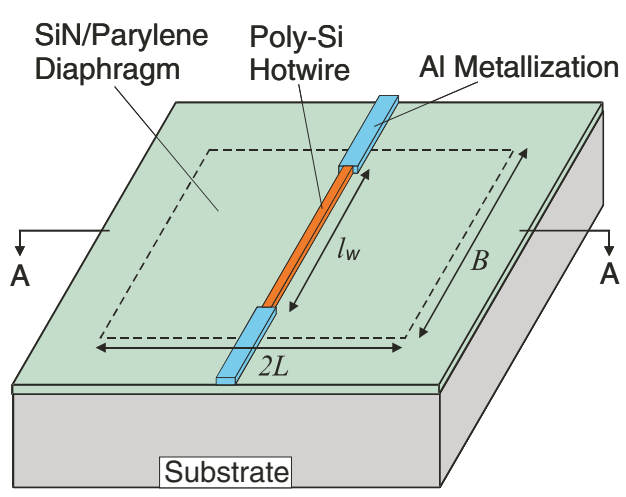

(a)

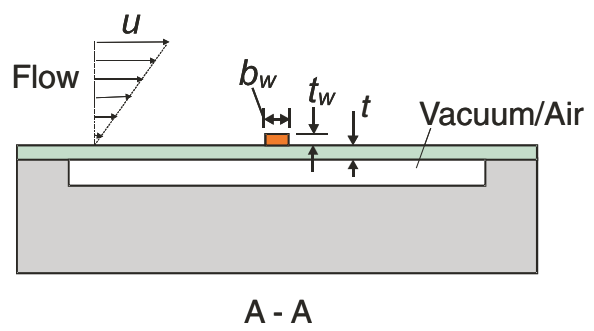

(b)

Figure 1. Schematic of MEMS thermal shear-stress sensors.

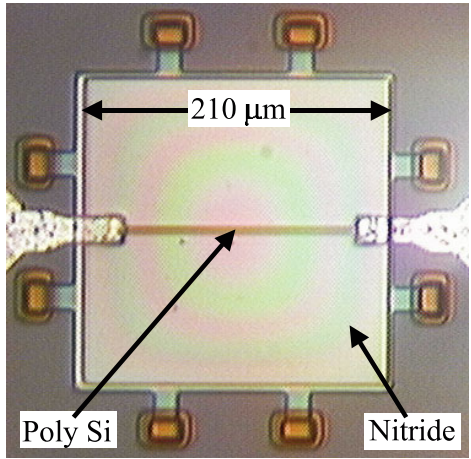

(a)

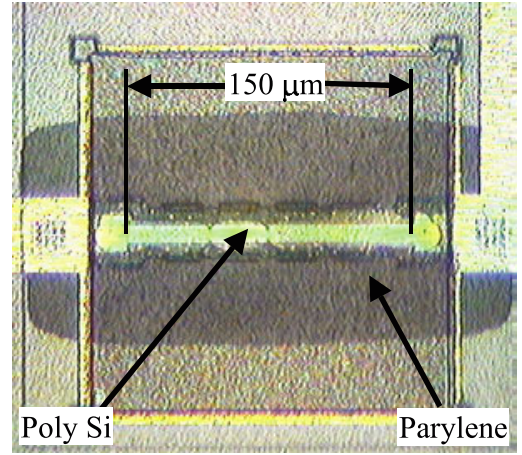

(b)

Figure 2. MEMS shear-stress sensors using $(a)$ nitride and $(b)$ Parylene diaphragms.

Table 1. Design dimensions (figure 1) and measured properties of the MEMS prototype shear-stress sensors used in wind-tunnel tests.

\begin{tabular}{lllll}
\hline & \multicolumn{3}{c}{ Sensor } \\
\cline { 2 - 5 } & Thin-wire & Thin-nitride & Thick-nitride & Parylene \\
\hline Property & & & \\
$2 L \times B \times t\left(\mu \mathrm{m}^{3}\right)$ & $210 \times 210 \times 1.5$ & $210 \times 210 \times 1.5$ & $210 \times 210 \times 3$ & $210 \times 90 \times 1.5$ \\
$l_{w} \times b_{w} \times t_{w}\left(\mu \mathrm{m}^{3}\right)$ & $150 \times 3 \times 0.5$ & $150 \times 7 \times 0.5$ & $150 \times 7 \times 0.5$ & $150 \times 10 \times 0.5$ \\
$R_{0}(\mathrm{k} \Omega)$ & 2.05 & 1.43 & 1.48 & 0.38 \\
$\alpha_{T}\left(10^{-3} /{ }^{\circ} \mathrm{C}\right)$ & 0.85 & 1.07 & 1.07 & 1 \\
$T_{w}-T_{\infty}\left({ }^{\circ} \mathrm{C}\right)$ & 118 & 93.5 & 93.5 & 100 \\
\hline
\end{tabular}

post-CMOS process [23]. The biasing circuitry was first fabricated at Mitel Semiconductor (now Zarlink Semiconductor) with a $2 \mu \mathrm{m}$ p-well double poly/double metal CMOS process. The hot-wire sensing element was formed using the gate polysilicon layer. The post-CMOS process began with the selective removal of the metal and dielectric layers in the sensor areas. A $1 \mu \mathrm{m}$ thick Parylene-N layer was then deposited at room temperature and patterned by oxygen plasma etching to open releasing holes. Next, bulk silicon underneath the polysilicon sensing element was removed by $\mathrm{BrF}_{3}$ through the releasing holes. A second layer of Parylene-N (2 $\mu \mathrm{m})$ was then deposited. Since Parylene deposition is conformal, about $0.5-1 \mu \mathrm{m}$ of Parylene was also deposited on the backside of the sensor diaphragm. Oxygen plasma etch back was performed to thin down the diaphragm and remove Parylene from the sensing element. A fabricated Parylene-based shear-stress sensor is shown in figure $2(b)$.

\section{Wind-tunnel experiments}

We have conducted wind-tunnel experiments with four prototype MEMS shear-stress sensors fabricated using the processes described above. Three of the sensors (figure 2(a)) had a polysilicon hotwire on a silicon-nitride diaphragm, and are here descriptively referred to as the 'thin-wire', 'thinnitride' and 'thick-nitride' sensors according to their relative hot-wire and diaphragm dimensions. In the fourth sensor, referred to as the 'Parylene' sensor, the polysilicon hot wire, reinforced by a $25 \mu \mathrm{m}$ wide dielectric layer, resided on a Parylene diaphragm (figure 2(b)). The specific design dimensions of these sensors are given in table 1 .

To ensure uniform testing conditions and consistent testing results, the chips carrying the four prototype sensors were placed on a single PC board, as shown in figure 3. The PC board was then flush-mounted on a specially made plug that fitted into the wall of a wind tunnel, with the hot wire perpendicular to the flow. The wind tunnel supplied a 


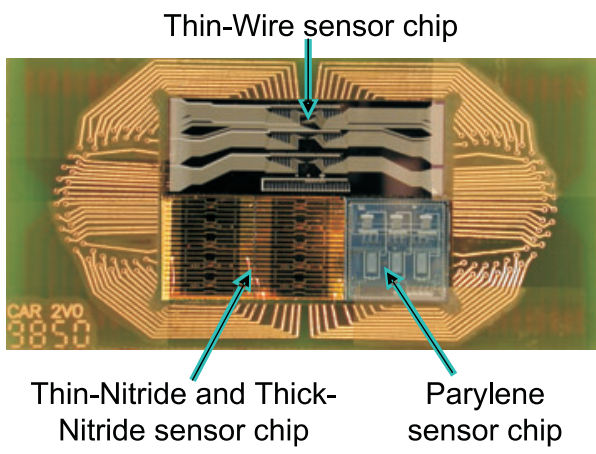

Figure 3. MEMS shear-stress sensors packaged on a single PC board.

two-dimensional channel flow [17]. The channel was $4.87 \mathrm{~m}$ long with a cross-sectional area of $60 \mathrm{~cm} \times 2.54 \mathrm{~cm}$. The channel walls were constructed of $2.5 \mathrm{~cm}$ thick Plexiglas and supported by a steel frame. An axial blower powered by a dc source supplied the air flow in the channel. At the highest blower speed, the centerline velocity in the channel was about $25 \mathrm{~m} \mathrm{~s}^{-1}$. Hotwire velocity measurements at $10 \mathrm{~m}$ $\mathrm{s}^{-1}$ indicated that the channel consisted of a laminar entrance flow region which gradually transformed into a fully developed turbulent flow in the downstream $2 / 3$ portion of the channel. All calibration and testing of the prototype sensors were carried out in this region of the channel. The wall shear stress was determined from the centerline velocity $U_{\mathrm{c}}$ in the channel using the correlation $\tau=\rho U_{t}^{2}$, where $U_{t}=0.1079 R e^{-0.089} U_{\mathrm{c}}$, $R e=U_{\mathrm{c}} d / v, \rho$ and $v$ are the air density and kinematic viscosity and $d$ is the half-height of the channel $(1.27 \mathrm{~cm})$ [17].

During testing, each sensor was biased with feedback control so that the resistive overheat ratio was maintained at $10 \%$, i.e., $\Delta R / R_{0}=0.1$, where $\Delta R=R-R_{0}$, and $R_{0}$ and $R$ are the hotwire resistances at ambient (or room) and operating temperatures $T_{\infty}$ and $T_{w}$, respectively. Therefore, the average hotwire temperature was maintained constant with $T_{w}-T_{\infty}=\left(\Delta R / R_{0}\right) / \alpha_{\mathrm{T}}$, where $\alpha_{\mathrm{T}}$ is the temperature coefficient of resistivity of the polysilicon hotwire. The values of $R_{0}, \alpha_{\mathrm{T}}$ and $T_{w}-T_{\infty}$ are listed in table 1. Fluctuations in ambient temperature were compensated with on-chip polysilicon thermistors.

The results from wind-tunnel testing of the prototype sensors are presented in figure 4. Here, $P$ is the hot-wire power corresponding to shear stress $\tau, P_{0}=\left.P\right|_{\tau=0}$ is the hotwire power with air still. Thus, $\left(P-P_{0}\right) / P_{0}$ is the relative net hot-wire power due to shear stress. The figure shows that the hot-wire power for the nitride-based sensors increases monotonically with shear stress. On the other hand, for the Parylene sensor, the hot-wire power initially exhibits a slight decrease with shear stress, and then monotonically increases. This unexpected phenomenon could be attributed to the piezoresistivity of polysilicon and needs to be further investigated. The experimental data from the four prototype sensors can be used to examine the validity of classical thermal shear-stress sensor theory, which states that the hot-wire power is proportional to the $1 / 3$-power of the shear stress. That is, $\left(P-P_{0}\right) / P_{0}$ plotted with respect to $\tau^{1 / 3}$ would appear as a straight line. This is clearly not true in figure 4 , suggesting that classical theory is not valid for the MEMS shear-stress sensors.

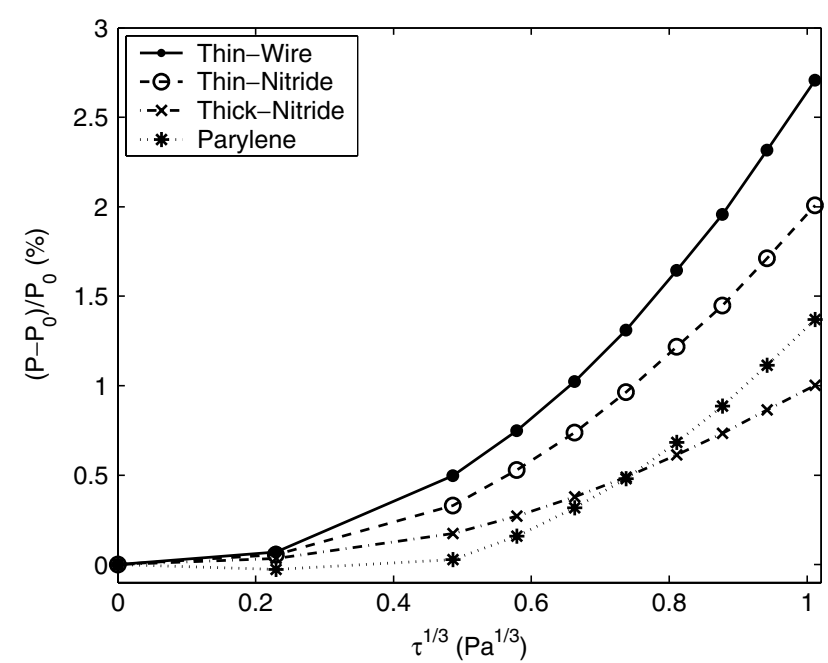

Figure 4. Shear-induced net hot-wire power versus $\tau^{1 / 3}$, measured from four prototype sensors.

\section{Inadequacy of classical theory for macroscale shear-stress sensors}

The classical shear-stress sensor theory [7] is examined in this section to elucidate the fundamental reason for its inadequacy when applied to MEMS sensors. This theory is concerned with a two-dimensional fluid flow over a plane surface. The temperature at the surface is at ambient $\left(T_{\infty}\right)$ except over a heated length $l=2 L$, where the temperature is $T_{w}$. The theory assumes that $\delta \ll l$ (or equivalently $\delta \ll L$ ), i.e., the thermal boundary layer (the heated region in the fluid) is thin compared to the heated length. In a coordinate frame with the $x$-axis on the surface and in the direction of the flow, and the $y$-axis perpendicular to the surface, the heat transfer in the flow is governed by the equation

$$
u \frac{\partial T}{\partial x}=\alpha \frac{\partial^{2} T}{\partial y^{2}},
$$

where $T$ is the fluid temperature and $\alpha$ the fluid's thermal diffusivity. Note that constant material properties are assumed throughout this paper. Under the assumption that the thermal boundary layer is also thin compared with the velocity boundary layer, the flow velocity $u$, which may vary with time, is given by the linear relationship [7]

$$
u=\frac{\tau y}{\mu},
$$

where $\mu$ is the fluid's dynamic viscosity. Solving this problem yields the following classical result:

$$
\frac{P-P_{0}}{k\left(T_{w}-T_{\infty}\right) B}=1.28\left(\frac{\tau L^{2}}{\alpha \mu}\right)^{1 / 3} .
$$

That is, the heating power transferred to the fluid over the heated length is proportional to $\tau^{1 / 3}$.

Here, it is important to note that the classical result is based on the assumption that the thermal boundary layer is thin compared with the heated length on the surface. From equations (1) and (2), the thermal boundary layer thickness $\delta$, defined as the vertical distance from the heated plane at which $\left(T-T_{\infty}\right) /\left(T_{w}-T_{\infty}\right)=0.01$, is found to be $\delta / L=$ $3.68\left(\tau L^{2} / \alpha \mu\right)^{-1 / 3}$. Thus, the thin thermal boundary 
Thick Thermal boundary layer

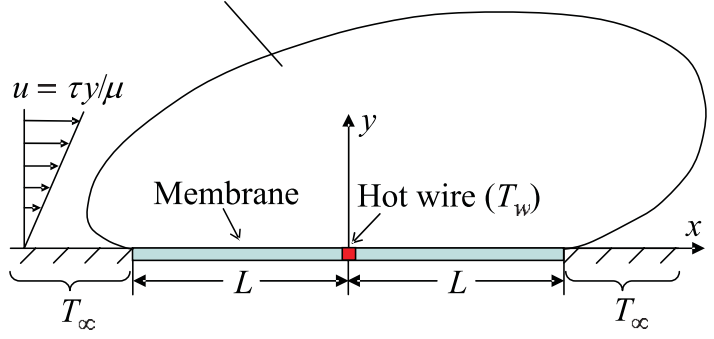

Figure 5. Two-dimensional MEMS shear-stress sensor model.

assumption $\delta / l \ll 1$ can be expressed in terms of shear stress as

$$
\frac{\tau L^{2}}{\alpha \mu} \gg 50 .
$$

Equation (4) is the condition that must be satisfied in order for the classical theory to be valid, and can be examined (with $L$ taken to be the diaphragm half-length) for the prototype MEMS sensors. With the thermophysical properties of air evaluated at the average of the hot-wire and ambient temperatures, we find that $\tau L^{2} / \alpha \mu<19.1$ for the nitridebased sensors, and $\tau L^{2} / \alpha \mu<3.42$ for the Parylene sensor. This indicates that the condition (4) is not satisfied, and the thermal boundary layer is not thin compared with the diaphragm length. As a result, the classical shear-stress theory is not valid for the MEMS sensors.

\section{Two-dimensional model for MEMS shear-stress sensors}

Given the inadequacy of the classical theory, this section presents a two-dimensional model for MEMS shear-stress sensors. By ignoring three-dimensional heat-transfer effects, this model allows simplifications that effectively reduce the problem complexity, but yet capture the essential characteristics of MEMS sensors. In particular, the model allows for thermal boundary layers that are comparable with sensor dimensions, and accounts for heat conduction in the diaphragm. Note that the inclusion of heat conduction in the diaphragm is important, as heat flux computations with abrupt changes in the prescribed surface temperature, as assumed in the classical model, are ill posed in the absence of a thin thermal boundary layer.

The two-dimensional MEMS sensor model is illustrated in figure 5. The heat-transfer problem considered involves forced convection in the fluid coupled with heat conduction in the diaphragm, which has length $2 L$. The diaphragm's midpoint is maintained at constant temperature $T_{w}$, representing the hotwire. This essentially ignores the hot wire's streamwise width, which is much smaller than $L$. In the coordinate frame shown in the figure, forced convection in the fluid is governed by [34]

$$
u \frac{\partial T}{\partial x}=\alpha\left(\frac{\partial^{2} T}{\partial x^{2}}+\frac{\partial^{2} T}{\partial y^{2}}\right),
$$

where the flow has been assumed to be either steady or vary with time at a sufficiently slow rate so that the heat transfer is in pseudo-steady state (i.e., the temperature field can be determined from steady-state governing equations) [7]. Contributions of natural convection and radiation to heat transfer have been ignored in this equation (see the discussion in section 6).

Comparing equations (1) and (5), we see that the classical theory ignores the contribution from streamwise heat conduction, which is important for MEMS sensors due to the lack of a thin thermal boundary layer. On the other hand, the profile of the flow velocity $u$ given by equation (2) is still valid due to the miniature sensor size. Heat conduction in the diaphragm, assumed to be in pseudo-steady state, is governed by

$$
k_{\mathrm{d}} t \frac{\partial^{2} T_{\mathrm{d}}}{\partial x^{2}}+\left.k \frac{\partial T}{\partial y}\right|_{y=0}=0,
$$

where $T_{\mathrm{d}}$ is the diaphragm temperature averaged over the thickness $t(t \ll L)$ and $k_{\mathrm{d}}$ is the diaphragm's thermal conductivity. The coupled heat-transfer problem is closed by the following boundary conditions: $T=T_{w}$ for $x=0$ and $y=0 ; T=T_{\infty}$ for $|x|>L$ and $y=0$ as well as for $x \rightarrow \pm \infty$ or $y \rightarrow+\infty$; and $T_{\mathrm{d}}=T$ as $|x| \leqslant L$ and $y=0$.

While the solution to this model is generally not in closed form, its functional form can be identified. Define three dimensionless parameters by

$$
\bar{\tau}=\frac{\tau L^{2}}{\alpha \mu}, \quad \bar{P}=\frac{P}{k\left(T_{w}-T_{\infty}\right) B}, \quad \text { and } \quad \lambda=\frac{k_{\mathrm{d}} t}{k L},
$$

where $B$ is the sensor's spanwise length (figure 1). It can be shown that the solution to the MEMS sensor model is given by the dimensionless power $\bar{P}$ as a function of the thermal conductivity ratio $\lambda$ and the dimensionless shear stress $\bar{\tau}$. That is, the solution has the functional form

$$
\bar{P}=\bar{P}(\lambda, \bar{\tau})=\bar{P}_{0}(\lambda)+\Delta \bar{P}(\lambda, \bar{\tau}),
$$

where we have decomposed the dimensionless power into two components: the hot-wire power in still air $\bar{P}_{0}=\bar{P}(\lambda, 0)$, and the shear-induced net hot-wire power $\Delta \bar{P}=\bar{P}-\bar{P}_{0}$.

Equations (5) and (6) can be solved numerically for the functional relationship (8), and the results are plotted in figure 6 . It can be seen from figure $6(a)$ that the still-air hot-wire power increases with the conductivity ratio $\lambda$. This is expected as the heat-transfer rates are higher for more thermally conducting diaphragms. The increase is nonlinear at small $\lambda$ values, and becomes highly linear as $\lambda$ further increases. The linearity can be explained by noting that when $\lambda$ is sufficiently large and the hot-wire temperature is held constant (with the sensor in constant-temperature mode), there is sufficient heat conduction in the diaphragm, so that the temperature distributions on the diaphragm surface become insensitive to the specific value of $\lambda$. The dependence of the dimensionless net hot-wire power $\Delta \bar{P}$ on dimensionless shear-stress $\bar{\tau}$ and conductivity ratio $\lambda$ is shown in figure $6(b)$. It can be seen that for a given $\lambda, \Delta \bar{P}$ always increases with $\bar{\tau}$, as expected from the fact that greater shear stress leads to higher heat-transfer rates. At a given $\tau, \Delta \bar{P}$ increases with $\lambda$, consistent with the fact that higher diaphragm conductivities allow for a larger effective heated region on the diaphragm surface and hence increased forced convection heat-transfer rates. However, the heat-transfer rate quickly becomes saturated as $\lambda$ further increases, and an asymptotic temperature distribution on the diaphragm is achieved. 


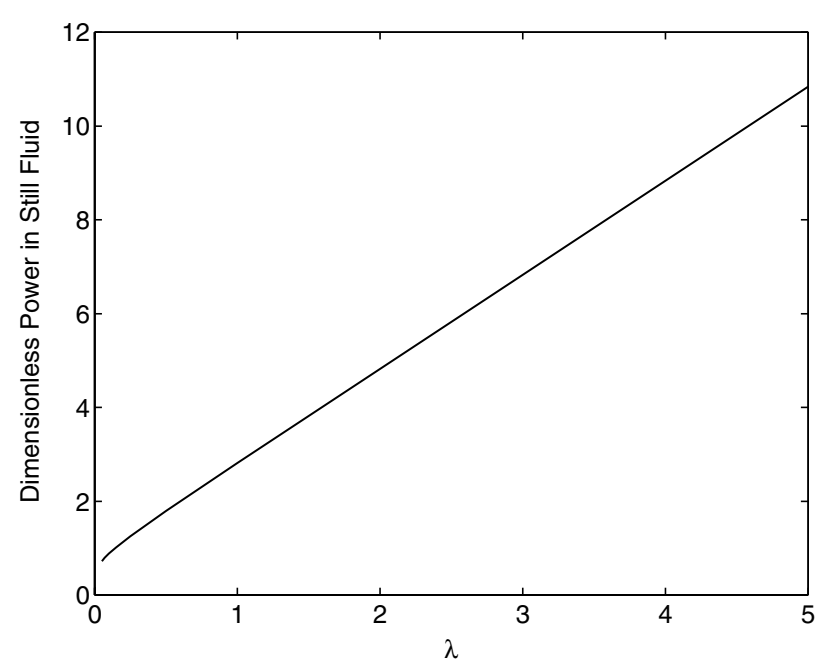

(a)

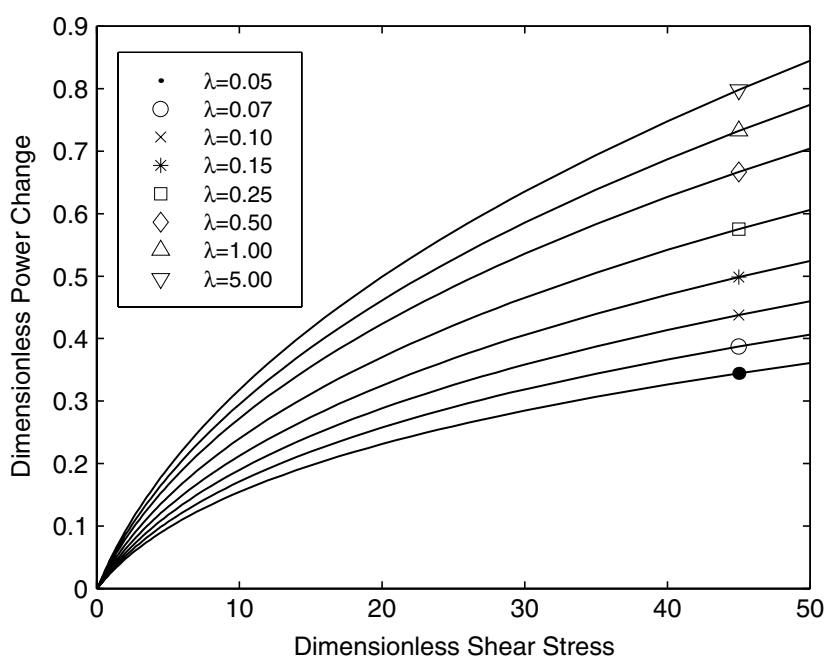

(b)

Figure 6. Numerical solution of the two-dimensional model: $(a)$ dimensionless hot-wire power in still air, and $(b)$ dimensionless net hot-wire power induced by shear stress.

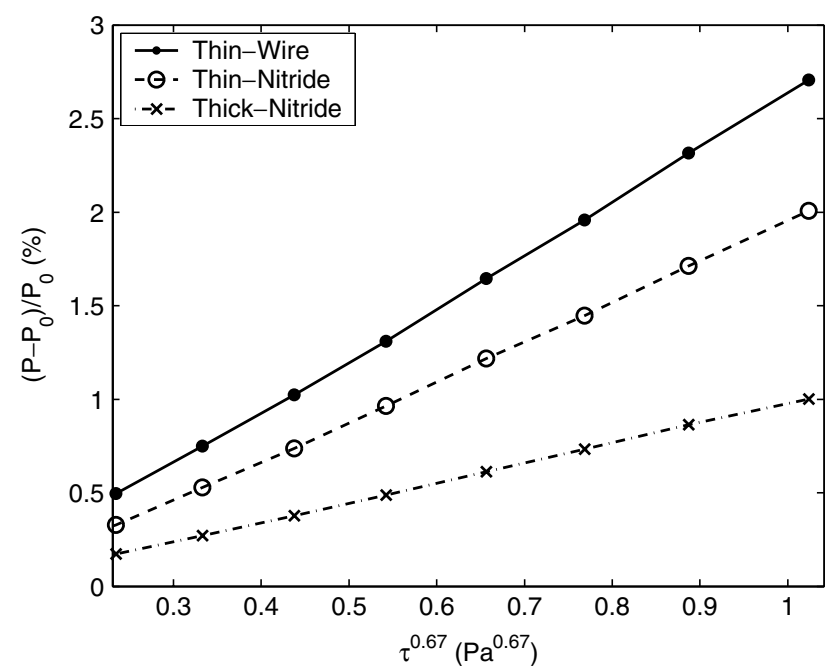

(a)

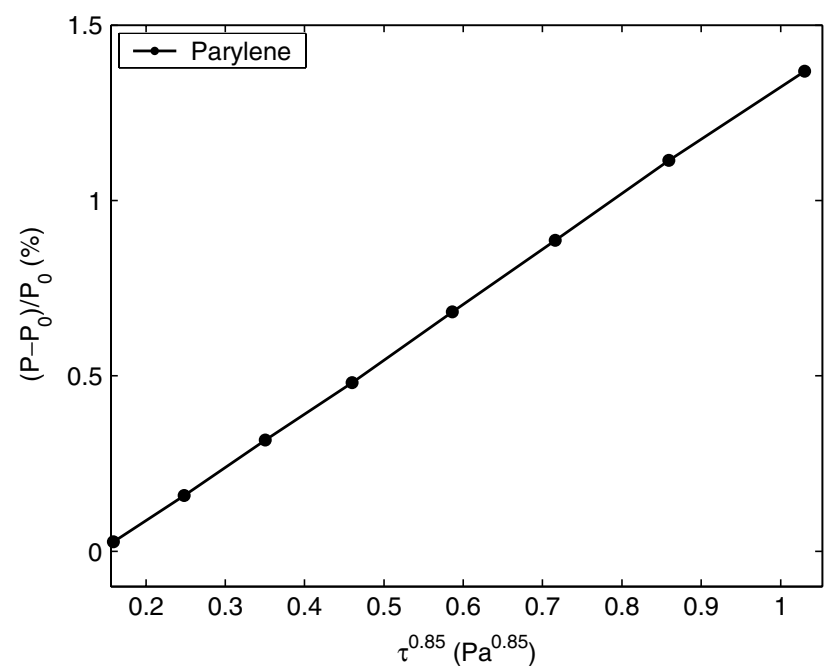

(b)

Figure 7. Experimentally determined dimensionless net hot-wire power versus $(a) \tau^{0.67}$ for the nitride-based sensors, and $(b) \tau^{0.85}$ for the Parylene sensor.

The numerical solution presented in figure 6 can be approximated by the following closed-form expressions. For $0.04 \leqslant \lambda \leqslant 5$ and $1 \leqslant \bar{\tau} \leqslant 50$, within $5 \%$ error we have

$$
\begin{aligned}
\bar{P}_{0} & =1.96 \lambda\left(1+0.435 / \lambda^{0.9}\right), \\
\Delta \bar{P} & =\frac{1.31 \lambda^{0.4} \bar{\tau}^{1.47}}{\left(1+2.07 \lambda^{0.6}\right)\left(1+0.56 \bar{\tau}^{0.3} / \lambda^{0.037}\right)^{5}} .
\end{aligned}
$$

These correlations provide practically useful design equations, and also suggest that the net hot-wire power in general is not a simple polynomial function of shear stress as predicted by classical theory. However, to gain further insight into the sensor behavior and the inadequacy of classical theory, the net hot-wire power can be approximated by polynomials in smaller shear-stress ranges. Specifically, for $2.5 \leqslant \bar{\tau} \leqslant 25$ (corresponding approximately to $0.15 \mathrm{~Pa} \leqslant \tau \leqslant 1.5 \mathrm{~Pa}$ for the nitride-based prototype sensors),

$$
\Delta \bar{P}=\left[0.187 \lambda^{0.5} /\left(1+2.1 \lambda^{0.59}\right)\right] \bar{\tau}^{0.67}
$$

and for $0.6 \leqslant \bar{\tau} \leqslant 5$ (or approximately $0.2 \mathrm{~Pa} \leqslant \tau \leqslant 1.5 \mathrm{~Pa}$ for the Parylene sensor),

$$
\Delta \bar{P}=\left[0.129 \lambda^{0.45} /\left(1+1.81 \lambda^{0.55}\right)\right] \bar{\tau}^{0.85} .
$$

Both of these correlations are obtained in the same range of conductivity ratio $0.04 \leqslant \lambda \leqslant 5$, and hold within $10 \%$ compared with numerical results.

The experimental data are plotted in figure 7 using the approximate power laws given in equations (10) and (11) within the appropriate shear-stress ranges. It can be seen that the net hot-wire power for the nitride-based sensors is indeed approximately proportional to the 0.67 th-power of shear stress (figure $7(a)$ ), and that for the Parylene also approximately follows the 0.85 th-power law (figure $7(b)$ ). This confirms that 
the two-dimensional shear-stress theory correctly captures the essential heat-transfer characteristics and predicts the trend in the MEMS sensor operation.

\section{Three-dimensional simulations of prototype MEMS shear-stress sensors}

The two-dimensional theory presented above offers valuable insight into the qualitative characteristics of the prototype MEMS shear-stress sensors. This section further seeks to understand the quantitative behavior of the protoype sensors by considering three-dimensional heat-transfer effects using a more accurate representation of the sensor configuration.

The three-dimensional MEMS sensor model is illustrated in figure 1 and consists of a polysilicon hot wire on a silicon nitride or Parylene diaphragm. The nitride diaphragm is thermally insulated from below by a vacuum cavity, and the Parylene diaphragm by an air cavity. The air cavity, which generally takes on an irregular shape, is approximated to have the shape of a rectangular block defined by the diaphragm length and width, with an estimated average thickness of $20 \mu \mathrm{m}$. Since silicon is an excellent thermal conductor, the substrate surface and the diaphragm edges are assumed to be at ambient temperature. This has been verified by measurements in which the substrate temperature in the immediate vicinity of the diaphragm was found to be no more than $1{ }^{\circ} \mathrm{C}$ above ambient, negligibly small compared with the sensors' operating temperature. The model considers heat conduction in the polysilicon hot wire, aluminum metallization and nitride or Parylene diaphragms. For the Parylene sensor, heat conduction in the air gap below the diaphragm is also included. Forced convection in the air flow above the diaphragm and hot wire is included in the model, assuming a linear velocity profile given in (2). The coupled conduction-convection heattransfer problem is solved numerically using the ABAQUS finite element code.

The effects of natural convection and radiation have been ignored in the three-dimensional simulations as well as in the two-dimensional parametrized model (section 5). The fluid flow induced by natural convection is characterized by the Grashof number $G r=g \beta\left(T_{w}-T_{\infty}\right) l^{3} / \nu^{2}$, where $l=2 L, g$ is the gravitational acceleration and $\beta$ is the fluid's volumetric thermal expansion coefficient (and $v$ is again the kinematic viscosity). To compare the relative significance of natural convection with respect to forced convection, the Reynolds number can be defined by $R e=(\tau L / \mu) L / \nu$ based on the fluid velocity at a distance $L$ above the diaphragm for shear stress $\tau$ to characterize the fluid flow associated with forced convection. For example, at $\tau \sim 0.3 \mathrm{~Pa}$, a relatively small shear stress, it can be calculated that $G r \sim 0.06$ and $\operatorname{Re} \sim$ 1.5 for the nitride-based sensors, and $G r \sim 0.005$ and $\operatorname{Re} \sim$ 0.3 for the Parylene sensor. Thus, the criterion $\mathrm{Gr} / \operatorname{Re}^{2} \ll 1$ is satisfied by all prototype sensors, and natural convection is negligible compared with forced convection [34]. As for natural convection effects above the diaphragm at small or zero shear stress, or below the diaphragm for the Parylenebased sensor, it can be shown from basic natural convection equations [34] that the relative contribution of natural convection to the total hot-wire power is represented by $G r^{1 / 2} v / \alpha<\sim 0.2$, and can therefore be reasonably ignored

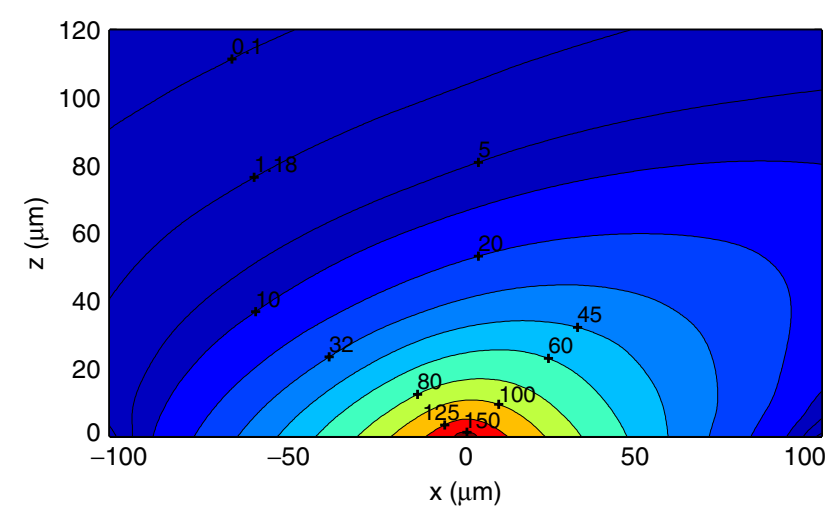

Figure 8. Calculated distribution of the air temperature $\left(T_{w}-T_{\infty}\right.$ in $\left.{ }^{\circ} \mathrm{C}\right)$ above the silicon nitride diaphragm of the thin-wire sensor at $\tau=1 \mathrm{~Pa}$.

considering the resulting significant simplification of the heattransfer problem.

The total radiation heat loss from the two sides of the diaphragm is estimated to be a few tens of microwatts in the worst-case scenario where the diaphragm surface emissivity is unity. This is about $1 \%$ of the still-air hot-wire power (of the order of milliwatts as given in table 1), but is comparable to the net hot-wire power exclusively contributed by shear stress. Fortunately, in the constant-temperature mode of sensor operation, the small shear-induced hot-wire power does not significantly change the temperature distribution of the diaphragm. As the total radiation heat loss is primarily determined by this temperature distribution, the total radiation heat loss should be largely independent of shear stress and cancel out in the shear-induced net hotwire power. The simplification of ignoring heat radiation in the model is thus acceptable.

The coupled conduction-convection heat-transfer problem has been solved using ABAQUS. In the simulations, the thermophysical properties of air are calculated at the average thermal boundary layer temperature $T_{a v}=\left(T_{w}+\right.$ $T_{\infty}$ ) $/ 2$ (with ambient temperature taken to be $T_{\infty}=25{ }^{\circ} \mathrm{C}$ ) from published data [34]. The thermal conductivities of polysilicon, silicon nitride, Parylene- $\mathrm{N}$ and aluminum are given nominal values $34,3.2,0.128$ and $237 \mathrm{~W} \mathrm{~m}^{-1} \mathrm{~K}^{-1}$, respectively. Figure 8 depicts the distribution of air temperature (relative to ambient temperature) in the streamwise vertical plane of symmetry for the thin-wire sensor (with nominal material properties and geometric dimensions), when operating at a constant average hot-wire temperature of $118{ }^{\circ} \mathrm{C}$ (see table 1) above room ambient at shear stress $\tau=1 \mathrm{~Pa}$. In the figure, the $x$-axis is on the diaphragm and aligned with the flow, while the $z$-axis is perpendicular to the diaphragm and points into the flow. The origin is at the center of the hot wire. It is customary to define the thermal boundary layer as the region in the air in which $\left(T-T_{\infty}\right) /\left(T_{w}-T_{\infty}\right)>0.99$. Thus, the thermal boundary layer is bounded by the level set $T-T_{\infty}=1.18^{\circ} \mathrm{C}$. It can be seen from the figure that this region extends far beyond a thin layer above the diaphragm. This confirms that a thin thermal boundary layer does not exist for the MEMS sensor and therefore the classical theory is inappropriate.

We now compare numerical simulation results with experimental data. When nominal material properties are used, 


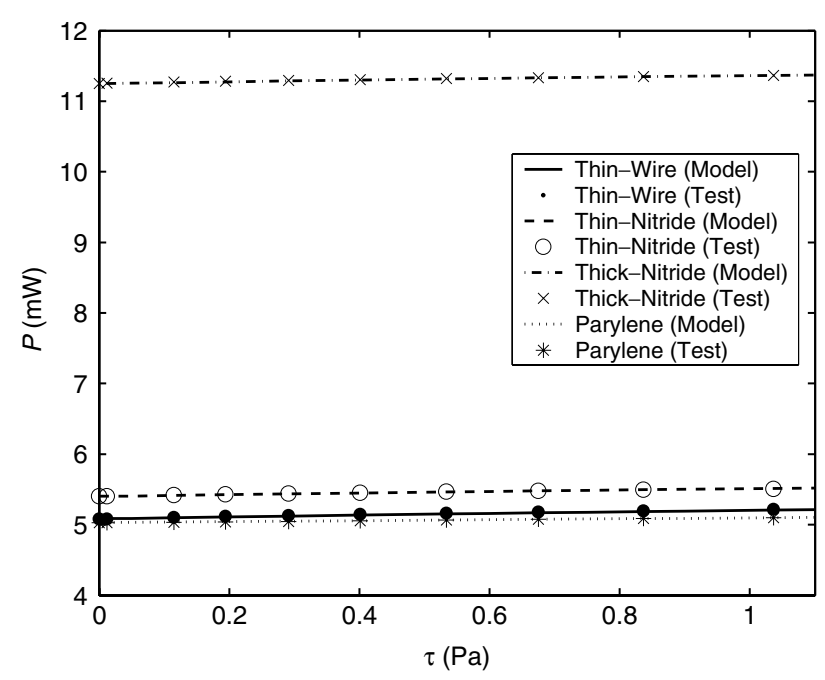

(a)

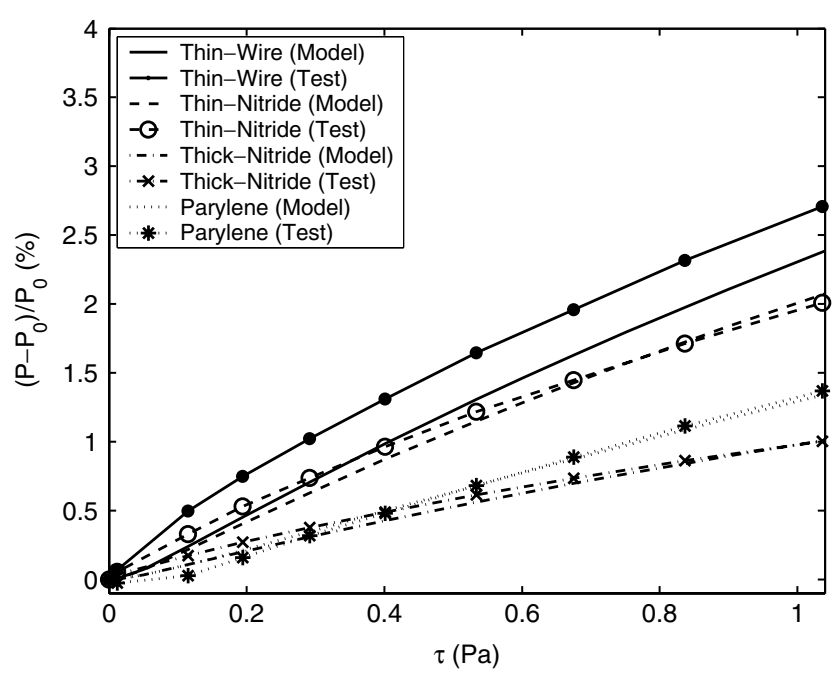

(b)

Figure 9. Comparison of simulation and experimental results: $(a)$ total hot-wire power, and $(b)$ relative net hot-wire power.

the simulations provide predictions with the correct order of magnitude, with relative errors of $1.6 \%, 16 \%, 34 \%$ and $10 \%$ as compared with experimental results for the four prototype sensors, respectively. This is satisfactory as it is known that the material properties are commonly process dependent and may significantly deviate from their nominal values, and that geometric dimensions of the sensors may also deviate from the design values due to die-to-die non-uniformities. These uncertainties can generally be manifested in the product of the diaphragm thermal conductivity and thickness. Hence, this product was allowed to be adjusted in the simulations to be $2 \%, 29 \%, 60 \%$ and $50 \%$ larger than the nominal values for the thin-wire, thin-nitride, thick-nitride and Parylene sensors in the simulations. The simulation results thus obtained are compared with the experimental data in figure 9. Excellent agreement can be observed for the total hot-wire power. As shown in figure $9(a)$, in a shear-stress range from 0 to $1 \mathrm{~Pa}$, relative errors between the simulation and experimentally determined hot-wire power are smaller than $0.2 \%$ for all four prototype sensors. This confirms that our three-dimensional model provides a valid quantitative description of the MEMS sensors tested.

The simulation results are further compared with the experimental data in terms of the net hot-wire power in figure $9(b)$. It can be seen that the net hot-wire power is a second-order quantity, accounting for less than $3 \%$ of the overall hot-wire power. Even for this small quantity, good agreement can be observed between the simulations and experiments, especially at relatively high shear-stress levels, where forced convection, the primary heat-transfer mode considered in the model, is most significant. The associated relative error is smaller than $3 \%$ for all prototype sensors except the thin-wire sensor, for which a relative error of $12 \%$ is observed. It is interesting to note that these errors in net hotwire power are insensitive to the choice of the material and geometric properties that are adjusted for the total hotwire power computations, and is essentially the same at a given shear stress even for the nominal values of these properties. The larger relative error for the thin-wire sensor could have been caused by the more significant temperature dependence of the heat-transfer problem, as this sensor operated at a temperature at least $20 \%$ higher than the other sensors. This needs to be further investigated using nonlinear heat transfer simulations.

\section{Conclusions}

An experimental and theoretical investigation of MEMS thermal shear-stress sensors has been presented. A set of microfabricated prototype shear-stress sensors that were based on silicon nitride or Parylene diaphragms were used to perform consistent shear-stress measurements in a wind tunnel to characterize the operating principle of such miniaturized devices. The testing results have confirmed that classical hot-wire shear-stress sensor theory, which states that the net hot-wire power is proportional to the 1/3-power of shear stress, is invalid for the MEMS sensors at shear-stress levels measured in the wind-tunnel experiments. To gain a fundamental understanding of this discrepancy, we carefully examined the validity conditions of the classical theory, and found that the inadequacy is caused by the lack of a thin thermal boundary layer at the MEMS sensor surface. We then presented a two-dimensional theory that considers heat conduction in the diaphragm as well as forced convection in the air flow. A dimensionless hot-wire power and a dimensionless shear stress are defined in the theory, and are related by closedform correlations that are parametrized by a dimensionless conductivity ratio. When applied to the prototype MEMS sensors, the theory predicts that the net hotwire power is approximately proportional to the 0.67 th and 0.85 th powers of the shear stress for the nitride-based sensors and the Parylene-based sensor, and these functional relationships correctly predict the trend in the experimental data. To obtain a quantitative understanding of the prototype sensors, simulations have also been performed using a more accurate, three-dimensional representation of the sensors. Excellent agreement is observed between the numerical simulations and experimental data. In particular, when material and 
geometric properties of the sensors are allowed to be adjusted to account for possible deviations of these properties from their nominal values, the total hot-wire power obtained from the simulations is within $0.2 \%$ of that determined experimentally for all prototype MEMS sensors. Satisfactory agreement for the net hot-wire power, a small second-order quantity that is exclusively due to shear stress and accounts for less than $3 \%$ of the total hot-wire power, has also been observed with a relative error smaller than $12 \%$.

The investigation presented in this paper captures the essential characteristics of MEMS thermal shear-stress sensors. There are, however, several issues that need to be further addressed. The first issue concerns unmodeled heattransfer effects, which include quantitative natural convection and radiation effects, as well as temperature dependence of material properties. In particular, it is well known that the thermophysical properties of air are quite sensitive to temperature. Over a temperature range of $100{ }^{\circ} \mathrm{C}$, these properties can vary from $20 \%$ to $70 \%$ [34]. While it was appropriate to use constant material properties as a first approximation, the inclusion of temperature-dependent material properties is expected to yield further accuracy improvements. Second, we have observed that the Parylene sensor exhibits a slight dip in hot-wire power at low shear stresses, and the reason for this phenomenon needs to be further investigated. Finally, the two-dimensional theory allows for insight into the general trend in the MEMS sensor behavior, but does not yet allow for quantitative evaluation of sensor characteristics, as three-dimensional heat-transfer effects that commonly exist in practical MEMS sensors are not included. While the three-dimensional simulations presented in this paper accurately account for these effects, they are not parametrized and cannot be reused if the sensor design is modified. Thus, further work needs to be conducted to develop parametrized, closed-form correlations, much like those for the two-dimensional theory presented in this paper, which accurately account for three-dimensional heat-transfer effects. Our work on developing such parametrized three-dimensional shear-stress sensor models will be reported in a separate publication.

\section{Acknowledgments}

The authors would like to thank Dr Steve Tung and Mr Chen-Wei Chiu for helpful discussions on MEMS shearstress sensor fabrication and testing. This work was supported by AFOSR under Grant F49620-97-1-0514.

\section{References}

[1] Kimura M, Tung S, Lew J, Ho C M, Jiang F and Tai Y C 1999 Measurements of wall shear stress of a turbulent boundary layer using a micro-shear-stress imaging chip Fluid Dyn. Res. 24 329- 42

[2] Bandyopadhyay P R 1994 Development of a microfabricated surface for turbulence diagnostics and control Application of Microfabrication to Fluid Mechanics (Chicago, IL) pp 67-74

[3] Bushnell D M 1983 Turbulent drag reduction for external flows AIAA Paper No 83-0227

[4] Moin P, Kim J and Choi H 1989 On the active control of wall-bounded turbulent flows AIAA Paper No 89-0960
[5] Ho C-M and Tai Y-C 1996 MEMS and its applications for flow control J. Fluids Eng. 118 437-47

[6] Haritonidis J H 1989 The measurements of wall shear stress Advances in Fluid Mechanics Measurements (Berlin: Springer) pp 229-61

[7] Goldstein S 1996 Fluid Mechanics Measurements 2nd edn (New York: Hemisphere)

[8] Schmidt M A, Howe R T, Senturia S D and Haritonidis J H 1988 Design and calibration of a microfabricated floatingelement shear-stress sensor IEEE Trans. Electron. Devices 35 750-7

[9] Shajii J, Ng K Y and Schmidt M A 1992 A microfabricated floating-element shear-stress sensor using wafer-bonding technology J. Microelectromech. Syst. 1 89-94

[10] Padmanabhan A, Goldberg H, Breuer K D and Schmidt M 1996 A wafer bonded floating element shear stress microsensor with optical position sensing by photodiodes J. Microelectromech. Syst. 5 307-15

[11] Pan T, Hyman D, Mehregany M, Reshotko E and Garverick S 1999 Microfabricated shear stress sensors: 1. Design and fabrication AIAA J. 37 66-72

[12] Hyman D, Pan T, Reshotko E and Mehregany M 1999 Microfabricated shear stress sensors: 2. Testing and calibration AIAA J. 37 73-8

[13] Brown G L 1967 Theory and application of heated films for skin friction measurements Proc. Heat Transfer Fluid Mech. Inst. pp 361-81

[14] Bellhouse B J and Schultz D L 1966 Determination of mean and dynamic skin friction, separation and transition in low-speed flow with a thin-film heated element J. Fluid Mech. 24 379-400

[15] Bellhouse B J and Schultz D L 1968 The measurement of fluctuating skin friction in air with heated thin-film gauges J. Fluid Mech. 32 675-80

[16] Ludwieg H 1950 Instrument for measuring the wall shearing stress of turbulent boundary layers Technical Memorandum No 1284 (Washington, DC: NACA)

[17] Liu C, Tai Y-C, Huang J B and Ho C-M 1994 Surface micromachined thermal shear stress sensor ASME Int. Mech. Eng. Congress and Exposition (Chicago, IL) pp 9-15

[18] Liu C, Huang J B, Zhu A, Jiang F, Tung S, Tai Y-C and Ho C-M 1999 A micromachined flow shear stress sensor based on thermal transfer principles J. Microelectromech. Syst. 8 90-9

[19] Jiang F, Tai Y-C, Huang J B and Ho C-M 1995 Polysilicon structures for shear stress sensors Proc. IEEE Region 10th Int. Conf. Microelectronics and VLSI (Hong Kong) pp 16-9

[20] Kalvesten E 1996 Pressure and wall shear stress sensors for turbulence measurements $P h D$ Thesis Royal Institute of Technology, Stockholm, Sweden

[21] Lofdahl L and Gad-el-Hak M 1999 MEMS-based pressure and shear stress sensors for turbulent flows Meas. Sci. Technol. 10 665-86

[22] Cain A, Chandrasekaran V, Nishida T and Sheplak M 2000 Development of a wafer-bonded, silicon-nitride membrane thermal shear-stress sensor with platinum sensing element Technical Digest, Solid-State Sensor and Actuator Workshop (Hilton Head Island, SC) pp 300-3

[23] Wang X Q, Han Z, Jiang F, Tsao T, Lin Q, Tai Y C, Koosh V, Goodman R, Lew J and Ho C M 1999 A fully integrated shear stress sensor Int. Conf. Solid-State Sensors and Actuators (Sendai, Japan) pp 1074-7

[24] Huang J B, Tung S, Ho C-M, Liu C and Tai Y-C 1996 Improved micro thermal shear-stress sensor IEEE Trans. Instrum. Meas. 45 570-4

[25] Sheplak M, Chandrasekaran V, Cain A, Nishida T and Cattafesta L N 2002 Characterization of a siliconmicromachined thermal shear-stress sensor AIAAJ. 40 1099-104

[26] Huang J B, Jiang F, Tai Y-C and Ho C-M 1999 A micro-electro-mechanical-system-based thermal 
shear-stress sensor with self-frequency compensation Meas. Sci. Technol. 10 687-96

[27] Jiang F, Tai Y-C, Gupta B, Goodman R, Tung S, Huang J B and Ho C-M 1996 A micromachined shear stress sensor imager Int. Workshop Micro Electro Mechanical Systems (San Diego, CA) pp 110-5

[28] Jiang F, Tai Y-C, Walsh K, Tsao T, Lee G B and Ho C-M 1997 A flexible MEMS technology and its first application to shear stress sensor skin Int. Workshop Micro Electro Mechanical Systems (Nagoya, Japan) pp 465-70

[29] Xu Y, Tai Y-C, Huang A and Ho C-M 2003 IC-integrated flexible shear-stress sensor skin J. Microelectromech. Syst. $12740-7$

[30] Tsao T, Jiang F, Miller R A, Tai Y-C, Gupta B, Goodman R, Tung S and Ho C-M 1997 An integrated MEMS system for turbulent boundary layer control Technical Digest, 1997 Int. Conf. on Solid State Sensors and Actuators (Chicago, IL) pp 315-8

[31] Xu Y, Jiang F, Lin Q, Clendenen J, Tung S and Tai Y-C 2002 Underwater shear-stress sensor Proc. IEEE Int. Conf. Micro Electro Mechanical Systems (Las Vegas, NV) pp 340-3

[32] Appukuttan A, Shyy W, Sheplak M and Cattafesta L 2003 Mixed convection induced by MEMS-based thermal shear stress sensors Numer. Heat Transfer A 43 283-305

[33] Meunier D, Tardu S, Tsamados D and Boussey J 2003 Realization and simulation of wall shear stress integrated sensors Microelectron. J. 34 1129-36

[34] Incropera F P and DeWitt D P 1996 Fundamentals of Heat and Mass Transfer 4th edn (New York: Wiley) 